\title{
Option Pricing with Bounded Expected Loss under Variance-Gamma Processes
}

\author{
Seongjoo Song ${ }^{1, a}$, Jongwoo Song ${ }^{b}$ \\ ${ }^{a}$ Department of Statistics, Korea University; ${ }^{b}$ Department of Statistics, Ewha Womans University
}

\begin{abstract}
Exponential Lévy models have become popular in modeling price processes recently in mathematical finance. Although it is a relatively simple extension of the geometric Brownian motion, it makes the market incomplete so that the option price is not uniquely determined. As a trial to find an appropriate price for an option, we suppose a situation where a hedger wants to initially invest as little as possible, but wants to have the expected squared loss at the end not exceeding a certain constant. For this, we assume that the underlying price process follows a variance-gamma model and it converges to a geometric Brownian motion as its quadratic variation converges to a constant. In the limit, we use the mean-variance approach to find the asymptotic minimum investment with the expected squared loss bounded. Some numerical results are also provided.
\end{abstract}

Keywords: Option pricing, variance-gamma processes, weak convergence, incomplete market, bounded loss.

\section{Introduction}

Lévy models with jumps have been frequently used as an alternative to the geometric Brownian motion in modeling the asset price process in mathematical finance during the last decade. Especially, exponential Lévy models with jumps of infinite activity, have become very popular. Madan and Seneta (1990), Eberlein and Keller (1995), Barndorff-Nielsen (1998) and Carr et al. (2002) are now well-known papers on Lévy modeling in mathematical finance among others. The main empirical motivation of using an exponential Lévy model comes from fitting the asset return distribution of real market data. With several particular choices for the Lévy process used in the exponential Lévy model, it is known that the model can account for some empirical features observed in real financial markets such as high kurtosis and nonzero skewness. As well as Lévy models are flexible enough to fit the distribution of historical data very accurately and explain the stylized empirical facts of time series of price process, (see Geman, 2002; Cont and Tankov, 2004 or Schoutens, 2003) they are also simple enough to develop varied mathematical theories of option pricing and hedging.

However, even though exponential Lévy models are relatively simple extensions of the BlackScholes model, they lead the market to an incomplete one. As well-known, the price of a contingent claim is not uniquely determined and the exact replication of an option is impossible in any incomplete market. Although there have been many attempts to find an appropriate price or an appropriate equivalent martingale measure, there is no universally accepted way of determining the price of a contingent claim. See Song and Song (2008) for some selected papers dealing with such attempts.

This research was supported by the Basic Science Research Program through the National Research Foundation of Korea (NRF) funded by the Ministry of Education, Science and Technology(No. 2009-0065128(S. Song) and 2010-0004196(J. Song)).

${ }^{1}$ Corresponding author: Associate Professor, Department of Statistics, Korea University, 5-1 Anam-dong, Sungbuk-gu, Seoul 136-701, Korea. E-mail: sjsong@korea.ac.kr 
With Lévy models, the characteristic function is explicitly expressed and option prices can be obtained using the inversion of the characteristic function if we can fix an appropriate equivalent martingale measure and estimate parameters under that measure. Indeed, the option pricing with Lévy models is typically studied in line of inversion, approximation and calibration. We can use the inversion formula to obtain the density function, write the expected value of the option payoff with the density, set it to be equal to the observed market price, calibrate the appropriate parameters, and then find the price of option of interest using the calibrated parameter values. However, this method needs to fix one equivalent martingale measure under which we calibrate the model, and typically needs some numerical methods such as Fast Fourier transform for inversion. Our pricing criterion does not use numerical approximation and does not require modeling under a specific martingale measure, although we utilize the minimal martingale measure in the limit as a tool to find a minimum possible investment.

The purpose of this paper is to find the minimum investment that guarantees the expected squared loss bounded by a certain level, as one attempt to find an appropriate price of an option in an incomplete market. The log of the underlying price process is assumed to follow the variance-gamma process, which is a relatively simple case of Lévy processes with jumps of infinite activity. More explicitly, we consider the sequence of variance-gamma processes that converges weakly to the BlackScholes model. This setup is the same as that of Song and Song (2008) where they consider the sequence of compound Poisson processes converging to the Black-Scholes model. It is also similar to that of Hong and Wee (2003) where they consider a sequence of jump-diffusion models that converges to the Black-Scholes model. In their paper, they priced a contingent claim using the minimal martingale measure and constructed the locally risk-minimizing strategy. They also studied the convergence of option prices as the underlying process converges to the Black-Scholes model.

Here, we consider the sequence of variance-gamma processes that converges weakly to the BlackScholes model, and try to find the minimum initial investment while the expected squared loss at the end is bounded by a given constant. The difference between Song and Song (2008) and this paper is the underlying asset price process. Compound Poisson processes considered in Song and Song (2008) have finite jump activity and are not enough to explain many of stylized empirical facts of real asset price processes. Here, we change the underlying process to a more realistic one and obtain similar results.

The remainder of the paper is organized as follows. Section 2 describes the detailed model and some weak convergence results. Section 3 studies the main problem by finding the mean-variance hedging strategy of the limit of the Black-Scholes hedging error and finding the minimum possible initial investment in the limit. We include some numerical results in Section 4. Section 5 contains concluding remarks.

\section{Model and Weak Convergences}

In many financial applications of Lévy models, the underlying stock price process, $S$, is assumed to follow

$$
S_{t}=S_{0} \exp \left(Z_{t}\right)
$$

where $Z=\left\{Z_{t}, t \geq 0\right\}$ is a Lévy process. A stochastic process $\left\{Z_{t}\right\}$ is a Lévy process if it has independent and stationary increments and stochastically continuous sample paths. $Z_{0}=0$, and it has random jump times. Lévy process includes Brownian motions, Poisson processes and compound Poisson processes as simple forms. 
One dimensional Lévy processes can be characterized by $\left(\sigma, v^{*}, \gamma\right)$, called as the characteristic triplet, or Lévy triplet of the process. With $\left(\sigma, v^{*}, \gamma\right)$, the characteristic function of $Z_{t}, E\left(e^{i u Z_{t}}\right)$ is represented as $e^{t \psi(u)}$ with the following Lévy -Khintchine formula,

$$
\psi(u)=i \gamma u-\frac{1}{2} \sigma^{2} u^{2}+\int_{-\infty}^{+\infty}\left(\exp (i u x)-1-i u x I_{\{|x|>1\}}\right) v^{*}(d x)
$$

where $\gamma \in \mathbb{R}, \sigma^{2} \geq 0$ and $v^{*}$ is a measure on $\mathbb{R} \backslash\{0\}$ with

$$
\int_{-\infty}^{+\infty}\left(1 \wedge x^{2}\right) v^{*}(d x)<\infty
$$

Lévy measure $v^{*}$ controls the jump dynamics of the process $Z$ so that jumps of sizes in the set $A$ occur according to a Poisson process with intensity parameter $\int_{A} v^{*}(d x)$. For more details on Lévy processes, especially used in the Finance area, one can refer to Cont and Tankov (2004) or Schoutens (2003).

When $Z=\left\{Z_{t}, t \geq 0\right\}$ follows a variance-gamma process with parameters $\sigma, v$ and $\theta$, the characteristic function of $Z_{t}$ will be

$$
\phi_{V G}(u ; \sigma, v, \theta)=\left(1-i u \theta v+\frac{1}{2} \sigma^{2} v u^{2}\right)^{-\frac{t}{v}} .
$$

Variance-gamma process can be represented as a time-changed Brownian motion with gamma subordinator as $Z_{t}=\theta G_{t}+\sigma W_{G_{t}}$, where $W$ is a Brownian motion and $G$ is a gamma process with parameters, $1 / v$ and $v$. It can also be represented as a difference of two gamma processes. When $G^{(1)}$ is a gamma process with parameters, $1 / v$ and $\sqrt{1 / 4 \theta^{2} v^{2}+1 / 2 \sigma^{2} v}+1 / 2 \theta v$, and $G^{(2)}$ is a gamma process with parameters, $1 / v$ and $\sqrt{1 / 4 \theta^{2} v^{2}+1 / 2 \sigma^{2} v}-1 / 2 \theta v$, we can see that $Z_{t}=G_{t}^{(1)}-G_{t}^{(2)}$.

Now, let us consider a sequence of variance-gamma processes that converges to a geometric Brownian motion. Each element of the sequence is a pure jump process, indexed by $\delta . \delta$ does not have a practical meaning, but it is used as a measure of the degree of discontinuity. A smaller $\delta$ means that the degree of discontinuity is smaller, i.e., the process is closer to a geometric Brownian motion.

For each of $\delta$, the log stock price process is defined on a probability space $\left(\Omega, \mathcal{F},\left(\mathcal{F}^{\delta}\right)_{0 \leq t \leq T}, \mathbf{P}\right)$ and follows

$$
\log S_{t}^{\delta}=\log S_{0}^{\delta}+Z_{t}^{\delta},
$$

where $\left\{Z_{t}^{\delta}\right\}$ follows is a variance-gamma process with parameters, $\sigma^{\delta}, v^{\delta}$ and $\theta^{\delta}$. As $\delta$ goes to 0 , we assume that $\theta^{\delta}$ converges to $\mu-1 / 2 \sigma^{2}$ for some constants $\mu$ and $\sigma, \sigma^{\delta}$ converges to $\sigma$, and $\nu^{\delta}$ converges to 0 . The filtration, $\left\{\mathcal{F}_{t}^{\delta}\right\}$, is generated by $S^{\delta}$ defined above, and we assume that the initial stock price $S_{0}^{\delta}$ is the same as $S_{0}$ for all $\delta$. As $\delta$ goes to 0 , the conditions on parameters above assure that $S^{\delta}$ converges weakly to a geometric Brownian motion.

Proposition 1. Assume all the above conditions. Then as $\delta$ goes to 0 , the process $\log S^{\delta}$ converges weakly to $\log S$ that is

$$
\log S_{t}=\log S_{0}+\left(\mu-\frac{1}{2} \sigma^{2}\right) t+\sigma B_{t},
$$

where B is a Brownian Motion under $\mathbf{P}$. 
Proof: See Appendix A.

Although the convergence in Proposition 1 holds when $\theta^{\delta} \rightarrow \mu-1 / 2 \sigma^{2}, \sigma^{\delta} \rightarrow \sigma$ and $\nu^{\delta} \rightarrow 0$, we will assume that $\theta^{\delta}=\mu-1 / 2 \sigma^{2}, \sigma^{\delta}=\sigma$ and $v^{\delta} \rightarrow 0$. For the application purpose, two sets of assumptions will not make much difference since we will estimate the values of $\mu$ and $\sigma$ from estimates of $\theta^{\delta}$ and $\sigma^{\delta}$. In fact, we could have some unidentifiability issue in the parameter estimation with the first set of assumptions.

We consider a market with two securities; a stock as a risky asset and a cash bond as a riskless asset. Then consider a European style option whose payoff is $H\left(S_{T}^{\delta}\right)$ with the expiration time $T$. We assume that $H\left(S_{T}^{\delta}\right)$ is in $L^{2}(\mathbf{P})$ and the interest rate $r$ is 0 without loss of generality so that the value of a unit of the cash bond is always 1. Throughout the paper, we denote $C(x, t)$ the solution of the Black-Scholes PDE at time $t<T$, with the terminal condition, $C(x, T)=H(x) . C_{S}, C_{S S}$ and $C_{S S S}$ denote the first, second, and third derivatives of $C(x, t)$ with respect to $x$, respectively. For each $\delta$, we compute $C\left(S_{t}^{\delta}, t\right)$ by plugging in the corresponding stock price process $S^{\delta}$. In other words, $C\left(S_{t}^{\delta}, t\right)$ is computed by the Black-Scholes PDE, but it may be different from what we observe from the market. On the other hand, $C\left(S_{t}, t\right)$ is also computed by the Black-Scholes PDE, but it is the true market price in the limit because the limiting stock price $S$ follows the geometric Brownian motion.

Let $X^{\delta}$ be the value process of the Black-Scholes hedging portfolio, i.e.,

$$
X_{t}^{\delta}=C\left(S_{0}^{\delta}, 0\right)+\int_{0}^{t} C_{S}\left(S_{u-}^{\delta}, u\right) d S_{u}^{\delta}
$$

In order to see the limit of the Black-Scholes hedging error, we define a sequence of numerical quantities, $\lambda_{\delta}$, that converges to $\infty$ as $\delta$ goes to 0 , representing the difference between the pre-limiting underlying process and the limiting process. For this, we define $\lambda_{\delta}$ as follows and use it to scale up the Black-Scholes hedging error in the following asymptotic procedures.

$$
\lambda_{\delta}=\left(\int z^{2} v^{* \delta}(d z)-\sigma^{2}\right)^{-1}\left(\int z v^{* \delta}(d z)\right)^{2},
$$

where $v^{* \delta}$ is the Lévy measure of the underlying variance-gamma process. This definition can be also used for more general Lévy models with suitable conditions. With the variance-gamma process that we now consider, $\lambda_{\delta}$ is the same as $1 / v^{\delta}$ and it goes to $\infty$ as $\delta$ goes to 0 by the previous assumption of $v^{\delta} \rightarrow 0 . \lambda_{\delta}$ can be seen as the reciprocal of the scaled difference of quadratic variations between pre-limiting and limiting processes.

Denote $R^{\delta}$ to be the scaled Black-Scholes hedging error, $\sqrt{\lambda_{\delta}}\left(C\left(S^{\delta}, \cdot\right)-X^{\delta}\right)$. Then we can show that $R^{\delta}$ converges to a continuous process jointly with $S^{\delta}$ in the following theorem.

Theorem 1. $\sqrt{\lambda_{\delta}}\left(C\left(S_{.}^{\delta}, \cdot\right)-X_{.}^{\delta}\right)$ converges jointly with $S^{\delta}$ in distribution to $(R, S)$ where

$$
R_{t}=\int_{0}^{t} \frac{\sqrt{3} \sigma^{2}}{2} S_{u}^{2} C_{S S}\left(S_{u}, u\right) d \tilde{W}_{u}
$$

and $\tilde{W}$ is a Brownian motion under $\mathbf{P}$ that is independent of $B$.

Proof: See Appendix B. 
Since we know that the scaled Black-Scholes hedging error $\sqrt{\lambda_{\delta}}\left(C\left(S^{\delta}, \cdot\right)-X^{\delta}\right)$ converges weakly to a continuous process $R$, a reasonable hedging strategy will have a form as

$$
X_{t}^{\delta}+\frac{1}{\sqrt{\lambda_{\delta}}} K_{t}^{\delta}=X_{t}^{\delta}+\frac{1}{\sqrt{\lambda_{\delta}}}\left(K_{0}+\int_{0}^{t} \eta_{u}^{\delta} d S_{u}^{\delta}\right)
$$

where $K_{0}$ is $\mathcal{F}_{0}$-measurable and $\eta_{u}^{\delta}$ is a hedging strategy for the Black-Scholes hedging error, $R^{\delta}$. We assume that $\eta^{\delta}$ is a predictable process with respect to $\left\{\mathcal{F}_{t}^{\delta}\right\}$. Assuming that $\eta^{\delta}$ converges weakly to $\eta$ jointly with $S^{\delta}$ and $R^{\delta}$ and $\eta$ is a predictable process with respect to $\left\{\mathcal{F}_{t}\right\}$, the limiting filtration generated by two Brownian Motions, $\tilde{W}$ and $B$, satisfying $E\left(\int_{0}^{T}\left(\eta_{u} S_{u}\right)^{2} d u\right)<\infty$, we can show that the Black-Scholes hedging error process, $R_{t}^{\delta}$, and $K_{t}^{\delta}=K_{0}+\int_{0}^{t} \eta_{u}^{\delta} d S_{u}^{\delta}$ converge jointly in distribution as follows.

Proposition 2. Assume the above conditions on $\eta^{\delta}$ and $\eta$ including that $\eta^{\delta} \stackrel{\mathcal{D}}{\longrightarrow} \eta$ jointly with $S^{\delta}$ and $R^{\delta}$. Then with $K_{t}=K_{0}+\int_{0}^{t} \eta_{u} d S_{u}$,

$$
\left(R^{\delta}, S^{\delta}, K^{\delta}\right) \stackrel{\mathcal{D}}{\longrightarrow}(R, S, K) .
$$

Proof: See Appendix C.

From now on, we will consider hedging strategies of the form in (2.5) only. The goal is to find the minimum possible investment with a bounded squared loss at the end by a fixed constant $U^{\prime}$ as

$$
E\left(H\left(S_{T}^{\delta}\right)-\left(X_{T}^{\delta}+\frac{1}{\sqrt{\lambda_{\delta}}} K_{T}^{\delta}\right)\right)^{2} \leq U^{\prime} .
$$

In other words, we want to find the smallest value of $K_{0}$ that satisfies

$$
E\left(R_{T}^{\delta}-K_{T}^{\delta}\right)^{2} \leq U_{\delta}
$$

for the upper bound $U_{\delta}=\lambda_{\delta} U^{\prime}$.

\section{Bounded Expected Loss}

In this section, we find the smallest possible investment with a bounded squared loss in the limit and based on the result in the limit, we propose a hedging strategy and the initial investment to use in the pre-limiting market. Since $\lambda_{\delta}$ is $1 / v^{\delta}$, we can calibrate the value of $\lambda_{\delta}$ from the market data. It means that $U_{\delta}$ in (2.6) can be determined as a constant before the asymptotics that will be studied below. Thus, throughout this section, we will drop the subscript $\delta$ from $U_{\delta}$.

It is easy to see that $\left(R_{T}^{\delta}-K_{T}^{\delta}\right)^{2}$ converges weakly to $\left(R_{T}-K_{T}\right)^{2}$ by the result from the previous section and continuity of the square function. However, as it is well-known, the convergence in distribution does not imply the convergence of expected values. If, for example, $R_{T}^{\delta}-K_{T}^{\delta}$ is uniformly integrable, $E\left(R_{T}^{\delta}-K_{T}^{\delta}\right)^{2}$ will be arbitrarily close to $E\left(R_{T}-K_{T}\right)^{2}$ for a small $\delta$, but we will not assume its uniform integrability in order to include more strategies under consideration. Nevertheless, here we find the smallest value $K_{0}$ in the limit with the constraint $E\left(R_{T}-K_{T}\right)^{2} \leq U$, along with the corresponding hedging strategy for $R_{T}, \eta$, in $K_{t}=K_{0}+\int_{0}^{t} \eta_{u} d S_{u}$. Then we use $K_{0}$ and the pre-limiting version of $\eta$ in the pre-limiting market and see the performance of this pair of initial investment and hedging strategy. 
Define $\Theta$ as a set $\left\{\eta\right.$ : predictable with respect to $\left.\mathcal{F}, E\left(\int_{0}^{T} \eta_{u}^{2} S_{u}^{2} d u\right)<\infty\right\}$. Note that if any pair $\left(K_{0}, \eta\right)$ solves the problem of minimizing $K_{0}$ subject to $E\left(R_{T}-K_{T}\right)^{2}=E\left(R_{T}-K_{0}-\int_{0}^{T} \eta_{u} d S_{u}\right)^{2} \leq U$, then $\left(K_{0}, \eta_{K_{0}}\right)$ also solves the same problem, where $\eta_{K_{0}}=\operatorname{argmin}_{\eta \in \Theta} E\left(R_{T}-K_{0}-\int_{0}^{T} \eta_{u} d S_{u}\right)^{2}$. So we can restrict our attention to a smaller set $\Theta^{\prime}$, which is $\left\{\eta \in \Theta: \eta \operatorname{minimizes} E\left(R_{T}-K_{0}-\int_{0}^{T} \eta_{u} d S_{u}\right)^{2}\right.$ for a certain value of $\left.K_{0}\right\}$, for a proper set of $\eta$ 's. We will consider only $\left\{K_{t}\right\}$ 's of the form $K_{t}=$ $K_{0}+\int_{0}^{t} \eta_{K_{0}, u} d S_{u}$. We denote $G_{t}(\eta)$ to be $\int_{0}^{t} \eta_{u} d S_{u}$.

Song and Song (2008) showed that the following proposition for a compound Poisson process as the underlying price process. (Proposition 3.1 and Proposition 3.2 in Song and Song (2008).) Since the model in Song and Song (2008) has the same limiting processes as in our set-up, the result in the limit will be the same. Thus, the proof for the following proposition is omitted.

Proposition 3. $\left\{K_{t}=K_{0}+\int_{0}^{t} \eta_{u} d S_{u}\right\}$ that makes the expected squared loss, $E\left(R_{T}-K_{T}\right)^{2}$, minimized for a given initial value $K_{0}$ is

$$
K_{t}=K_{0}+\int_{0}^{t} \frac{\mu}{\sigma^{2} S_{u}}\left(\frac{S_{u}}{S_{0}}\right)^{-\frac{\mu}{\sigma^{2}}} e^{-\frac{1}{2}\left(\frac{\mu^{2}}{\sigma^{2}}+\mu\right) u}\left(\int_{0}^{u}\left(\frac{S_{v}}{S_{0}}\right)^{\frac{\mu}{\sigma^{2}}} e^{\frac{1}{2}\left(\frac{\mu^{2}}{\sigma^{2}}+\mu\right) v} Y_{v} d \tilde{W}_{v}-K_{0}\right) d S_{u},
$$

where $Y_{u}=\sqrt{3} \sigma^{2} / 2 S_{u}^{2} C_{S S}\left(S_{u}, u\right)$ and $\tilde{W}_{u}$ is the Brownian motion introduced in Theorem 1. Moreover, the minimum investment subject to $E\left(R_{T}-K_{0}-G_{T}(\eta)\right)^{2} \leq U$ is

$$
\tilde{K}_{0}=-e^{\frac{\mu^{2}}{2 \sigma^{2}} T} \sqrt{U-\int_{0}^{T} E\left(e^{-\frac{\mu^{2}}{\sigma^{2}}(T-u)} Y_{u}^{2}\right) d u}
$$

If $U$ is smaller than $\int_{0}^{T} E\left(e^{-\mu^{2} / \sigma^{2}(T-u)} Y_{u}^{2}\right) d u$, it is not possible to make the expected squared loss bounded by $U$ and we need to increase the upper bound of the expected loss. When the contingent claim being considered is a call option, we can get a more explicit expression. If the option is a European call with the strike price $K$ and expiration time $T$, then the minimum investment will be

$$
\tilde{K}_{0}=-e^{\frac{\mu^{2}}{2 \sigma^{2}}} T \sqrt{\left(U-\frac{3 \sigma^{2}}{8 \pi} \int_{0}^{T} \frac{1}{\sqrt{T^{2}-u^{2}}} \exp \left(\frac{B(T)+C(T) u}{\sigma^{2}(T+u)}\right) d u\right)}
$$

where $B(T)=-\left(\log \left(S_{0} / K\right)\right)^{2}+\sigma^{2} T \log \left(S_{0} K\right)-\sigma^{4} / 4 T^{2}-\mu^{2} T^{2}$ and $C(T)=\sigma^{2} \mu T+2\left(\mu+\sigma^{2}\right) \log K-$ $2 \mu \log S_{0}$. For more details, see Song and Song (2008).

Let us use the pre-limiting version of what we have found in the pre-limiting market. The initial investment and the hedging strategy we would use generate the value of the portfolio as follows.

$$
\begin{aligned}
L_{t}^{\delta}= & X_{t}^{\delta}+\frac{1}{\sqrt{\lambda_{\delta}}}\left(\tilde{K}_{0}+\int_{0}^{t} \eta_{\tilde{K}_{0}, u}^{\delta} d S_{u}^{\delta}\right) \\
= & C\left(S_{0}, 0\right)+\int_{0}^{t} C_{S}\left(S_{u-}^{\delta}, u\right) d S_{u}^{\delta}+\frac{\tilde{K}_{0}}{\sqrt{\lambda_{\delta}}} \\
& +\frac{1}{\sqrt{\lambda_{\delta}}} \int_{0}^{t} \frac{\mu}{\sigma^{2} S_{u-}^{\delta}}\left(\frac{S_{u-}^{\delta}}{S_{0}}\right)^{-\frac{\mu}{\sigma^{2}}} e^{-\frac{1}{2}\left(\frac{\mu^{2}}{\sigma^{2}}+\mu\right) u} \\
& \times\left(\int_{0}^{u}\left(\frac{S_{v-}^{\delta}}{S_{0}}\right)^{\frac{\mu}{\sigma^{2}}} e^{\frac{1}{2}\left(\frac{\mu^{2}}{\sigma^{2}}+\mu\right) v} d R_{v}^{\delta}-\tilde{K}_{0}\right) d S_{u}^{\delta} .
\end{aligned}
$$


Table 1: Initial investment and $\sqrt{\text { MSHE}}$. When $\lambda_{\delta}=10,000$, we compare two cases of using Black-Scholes option price and hedging strategy and using $\tilde{K}_{0}$ and corresponding variance-gamma hedging strategy. We gain at the beginning with smaller initial investment and lose at the end with larger $\sqrt{\mathrm{MSHE}}$, but the loss is smaller than the gain.

\begin{tabular}{c|cc}
\hline \hline & Black-Scholes & Variance-gamma with $\tilde{K}_{0}$ \\
\hline initial investment (in \$) & 1.5717 & 1.4708 \\
$\sqrt{\mathrm{MSHE}}$ (in \$) & 0.065 & 0.105 \\
\hline \hline
\end{tabular}

The initial investment with the bounded loss constraint will be the Black-Scholes price, $C\left(S_{0}, 0\right)$, plus $\tilde{K}_{0} / \sqrt{\lambda_{\delta}}$. As we saw in Proposition $3, \tilde{K}_{0}$ is always negative so that our initial investment is smaller than the Black-Scholes price. By Song and Mykland (2006), $K_{0}=0$ minimizes $E\left(R_{T}-K_{0}-G_{T}\left(\theta_{K_{0}}\right)\right)^{2}$ among all possible values of $K_{0}$. Since we are willing to tolerate a higher level of error at the expiration in this case, we should be able to reduce the initial investment from the Black-Scholes price, and it has achieved by Proposition 3. In later sections, we will call $\eta_{\tilde{K}_{0}}$ and $\left\{L_{t}^{\delta}\right\}$ above as the variance-gamma hedging strategy and the value of the variance-gamma hedging portfolio, respectively.

\section{Numerical Results}

Consider a European call option that expires in 6 months. The interest rate is assumed to be $0, \mu$ is set to be 0.15 per annum, and $\sigma$ is set to be 0.2 per annum. We try $\lambda_{\delta}=10,000$. It means that the prelimiting variance-gamma process has the parameter values as $\theta^{\delta}=0.13, \sigma^{\delta}=0.2$ and $v^{\delta}=0.0001$. We set the strike price $K=\$ 60$ and the initial stock price $S_{0}=\$ 60$.

We rebalance the hedging portfolio in every .0001 years, approximately once in an hour and the number of generated sample paths is 1,000 . The Black-Scholes initial price is $\$ 1.5717$, in this case.

The simulation study is to compare the Black-Scholes portfolio with the variance-gamma hedging portfolio given in (3.1). The initial investment of Black-Scholes portfolio is $C\left(S_{0}, 0\right)$, and that of variance-gamma portfolio is $C\left(S_{0}, 0\right)+\tilde{K}_{0} / \sqrt{\lambda_{\delta}}$. We compare these values numerically and also compare the performance of two hedging portfolios by calculating their mean squares of hedging errors (abbreviated by MSHE). By hedging error, we mean the option payoff subtracted by the value of the hedging portfolio at the expiration. For example, the MSHE of the Black-Scholes strategy is

$$
E\left(\text { option payoff at the expiration }-C\left(S_{0}, 0\right)-\int_{0}^{T} C_{S}\left(S_{t-}^{\delta}, t\right) d S_{t}^{\delta}\right)^{2} .
$$

Suppose that we want the expected squared error loss at the end to be at most $U^{\prime}=0.01$. It means that the upper bound, $U$, in (2.6) is 100. The results of numerical integration and simulation with 1000 sample paths are given in Table 1 . By using the variance-gamma strategy with $\tilde{K}_{0}$, we gain 10 cents at the beginning over the case when we follow the Black-Scholes strategy, while we lose 4 cents in the square root of MSHE at the end. The loss at the end in the pre-limiting case is less than the gain at the beginning. Figure 1 compares densities of hedging errors for two hedging portfolios. We can see that the variance-gamma hedging error has a bit less variability than the Black-Scholes hedging error, although it has larger bias. The bias mainly comes from the lower initial investments.

The square root of the MSHE of the variance-gamma strategy with $\tilde{K}_{0}$ is supposed to be no more than 0.1 , the bound that we hoped to achieve, but in the simulation result, it is a bit larger than the upper bound. This discrepancy can be possibly explained by the fact that the computed MSHE is not a quantity in the limit. To see the distribution of the square root of the MSHE before the limit for other cases, we ran 1,000 simulations for $\lambda_{\delta}=5,000,10,000$ and 20,000. Each simulation has 1,000 


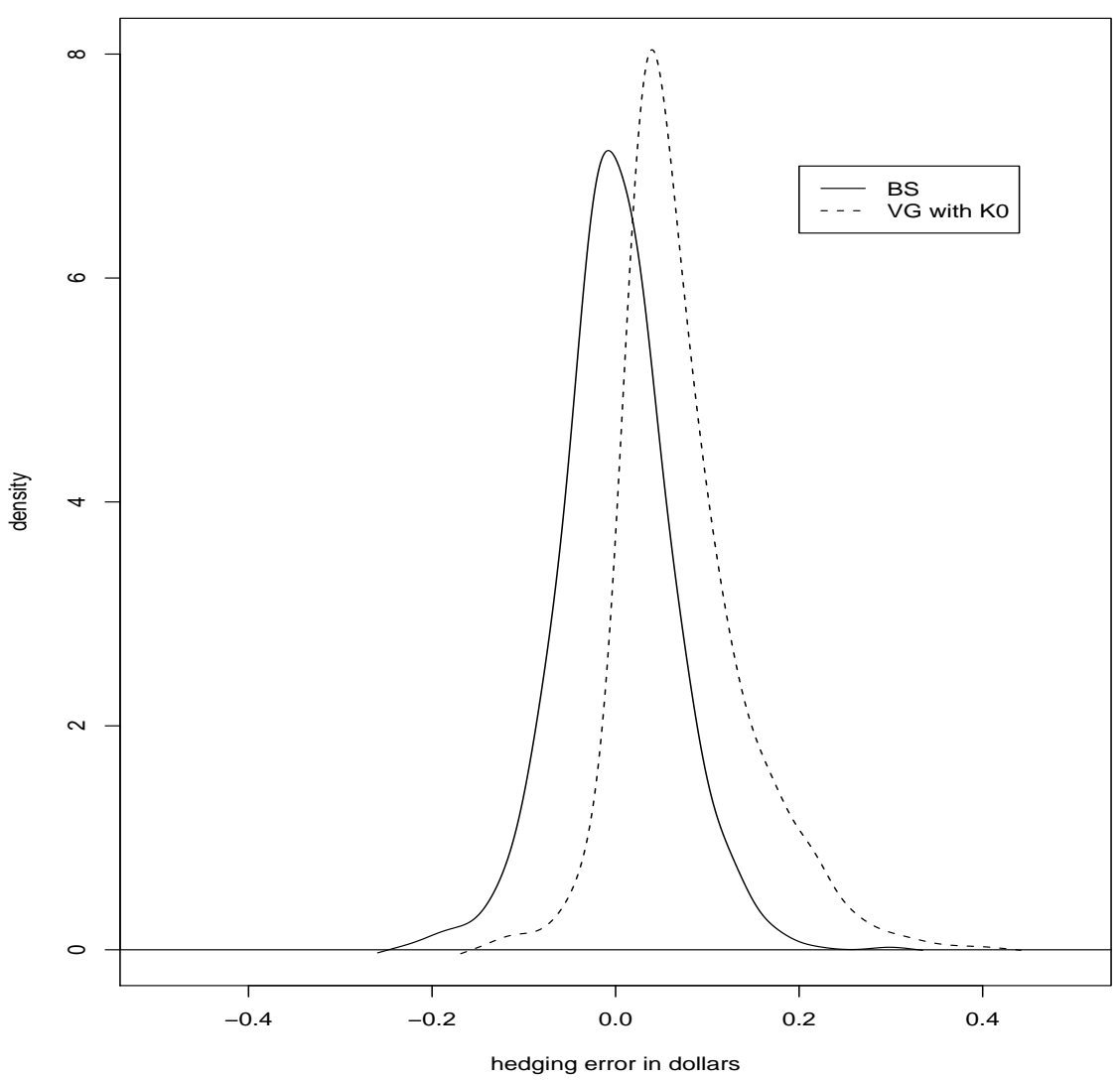

Figure 1: Comparison of densities of hedging errors. Full curve is for the error of the Black-Scholes hedging strategy and thr broken curve is for the error of the variance-gamma hedging strategy. $\lambda_{\delta}$ is 10,000 . The variancegamma hedging error has a bit less variability than the Black-Scholes hedging error but a larger bias due to the lower initial investment.

sample paths and we obtain one value of the square root of the MSHE from each simulation. Table 2 summarizes the result.

As expected, we see in Table 2 that when $\lambda_{\delta}$ increases, the values of MSHE of Black-Scholes strategy tend to decrease. As $\delta$ gets smaller, the Black-Scholes hedging strategy gets closer to the perfect hedging strategy. But the values of MSHE of variance-gamma strategy do not decrease much, since we aimed to minimize the initial investment with the bounded expected squared loss. In that sense, the MSHEs are supposed to have similar values close to the upper bound. Also, as seen in Table 1, the square root of the MSHE of the variance-gamma strategy is still larger than 0.1, but the values do not blow up as $\lambda_{\delta}$ increases and values are very close to the upper bound that we desired. The values of $\tilde{K}_{0} / \sqrt{\lambda_{\delta}}$ tell us how much we can reduce the initial investment from the Black-Scholes price by using the variance-gamma strategy. The values of $\tilde{K}_{0} / \sqrt{\lambda_{\delta}}$ do not decrease as $\lambda_{\delta}$ increases, and it is explained by the fact that the leading term of $\tilde{K}_{0}$ is $\exp \left(-\mu^{2} /\left(2 \sigma^{2}\right) T\right) \sqrt{\lambda_{\delta} U^{\prime}}$ from Proposition 3. The leading term of $\tilde{K}_{0} / \sqrt{\lambda_{\delta}}$ does not depend on $\delta$. From Table 2 , we can see that we still gain more at the beginning than the loss at the expiration by using the variance-gamma strategy, keeping the MSHE not too far from the original upper bound. 
Table 2: $\sqrt{\text { MSHE }}$ values for various $\lambda_{\delta}$ 's. For several different $\lambda_{\delta}$ values, we computed the $\sqrt{\text { MSHE }}$ of two pairs of initial investment and corresponding hedging strategies. The column names as $\tilde{K}_{0} / \sqrt{\lambda_{\delta}}$ shows how much we can save at the beginning by using $\tilde{K}_{0}$.

\begin{tabular}{c|ccc}
\hline \hline & $\tilde{K}_{0} / \sqrt{\lambda_{\delta}}$ & Black-Scholes & Variance-gamma with $\tilde{K}_{0}$ \\
\hline$\lambda_{\delta}=5,000$ & -0.084 & 0.082 & 0.106 \\
$\lambda_{\delta}=10,000$ & -0.101 & 0.065 & 0.105 \\
$\lambda_{\delta}=20,000$ & -0.108 & 0.054 & 0.105 \\
\hline \hline
\end{tabular}

\section{Concluding Remark}

In this paper, we have provided a method of finding a reasonable option price when the log of the asset price follows a variance-gamma model that converges to a Black-Scholes model. We have studied the problem assuming that the hedger wants to minimize the initial investment with the expected squared loss bounded by a constant.

We showed that the Black-Scholes hedging error, $R^{\delta}$, converges weakly to a continuous process, $R$, and found the mean-variance hedging strategy of $R_{T}$ in the limit. We also found the initial investment to achieve $E\left(R_{T}-K_{T}\right)^{2} \leq U$, where $K$ is the value process of the mean-variance hedging strategy of $R_{T}$ with a given initial value. Then we proposed to use the same initial investment and follow the prelimiting version of the mean-variance hedging strategy in the pre-limiting market, as given in (3.1). The corresponding hedging strategy to the smallest initial investment has a form of the Black-Scholes hedging strategy plus a correction term.

Numerical study shows that the reduction we achieved in the initial investment is more than the loss we have to endure at the expiration for several different $\lambda_{\delta}$ values. It means that what we have shown in the limiting market still holds in the pre-limiting market with finite $\lambda_{\delta}$ 's. Although the value of MSHE is a little bit larger than the upper bound that we set up in the beginning, there exists a solid gain over the case where we use the Black-Scholes hedging strategy in using the variance-gamma strategy with $\tilde{K}_{0}$.

\section{Appendix A: Proof of Proposition 1}

We assume that $\theta^{\delta}=\mu=1 / 2 \sigma^{2}, \sigma^{\delta}=\sigma$ and $\nu^{\delta} \rightarrow 0$ as $\delta \rightarrow 0$. Define $M^{\delta}$ and $M_{\epsilon}^{\delta}$ to be the martingale part of $\log S^{\delta}$ and the martingale part of $\log S^{\delta}$ with jump size greater than $\epsilon$ in absolute value, respectively. Then $M^{\delta}$ is a square-integrable martingale and

$$
\begin{aligned}
& \left\langle M^{\delta}, M^{\delta}\right\rangle_{t}=\int_{0}^{t} \int z^{2} v^{* \delta}(d z) d s=\left(\left(\theta^{\delta}\right)^{2} v^{\delta}+\left(\sigma^{\delta}\right)^{2}\right) t \underset{\delta \rightarrow 0}{\longrightarrow} \sigma^{2} t, \\
& \left\langle M_{\epsilon}^{\delta}, M_{\epsilon}^{\delta}\right\rangle_{t}=\int_{0}^{t} \int_{|z|>\epsilon} z^{2} v^{* \delta}(d z) d s \underset{\delta \rightarrow 0}{\longrightarrow} 0,
\end{aligned}
$$

where $v^{* \delta}$ is the Lévy measure of the underlying process as in (2.3). The above convergence results can easily be shown by plugging in the Lévy measure of the variance-gamma process. By Rebolledo's theorem (Andersen et al., 1992), $M^{\delta} \rightarrow \sigma B$ where $B$ is a Brownian motion under the limiting measure P, and thus, by Proposition VI.3.17 in Jacod and Shiryaev (1987),

$$
\log S^{\delta} \stackrel{\mathcal{D}}{\longrightarrow} \log S
$$




\section{Appendix B: Proof of Theorem 1}

Lemma 1. Define $\xi_{t}^{\delta}=\sqrt{\lambda_{\delta}}\left(\left[\log S^{\delta}, \log S^{\delta}\right]_{t}-\sigma^{2} t\right)$ and $\xi_{t}=\sqrt{3} \sigma^{2} \tilde{W}_{t}$. W is a Brownian motion that is independent of $B$, the Brownian motion that drives the limiting asset price process. Then,

$$
\left(\log S^{\delta}, \xi^{\delta}\right) \stackrel{\mathcal{D}}{\longrightarrow}(\log S, \xi) .
$$

Proof: Define $\hat{M}_{t}^{\delta}$ to be $\sqrt{\lambda_{\delta}}\left(\left[\log S^{\delta}, \log S^{\delta}\right]_{t}-\left\langle\log S^{\delta}, \log S^{\delta}\right\rangle_{t}\right)$. Then $\xi_{t}^{\delta}=\hat{M}_{t}^{\delta}+\sqrt{\lambda_{\delta}}\left(\int z^{2} v^{* \delta}(d z)-\right.$ $\left.\sigma^{2}\right) t=\hat{M}_{t}^{\delta}+o(1)$. Thus, it suffices to show that

$$
\left(\log S^{\delta}, \hat{M}^{\delta}\right) \stackrel{\mathcal{D}}{\longrightarrow}(\log S, \xi)
$$

and again, it is sufficient to show that

$$
\left(\log S^{\delta}-\log S_{0}-\theta^{\delta} t, M^{\delta}\right) \stackrel{\mathcal{D}}{\longrightarrow}(\sigma B, \xi)
$$

because $\log S_{t}=\log S_{0}+\left(\mu-1 / 2 \sigma^{2}\right) t+\sigma B_{t}$ and $\theta^{\delta}=\mu-1 / 2 \sigma^{2}$.

As in Appendix A, $M^{\delta}$ denotes the martingale part of $\log S^{\delta}$. Then $M^{\delta}$ and $\hat{M}^{\delta}$ are squareintegrable martingales satisfying

$$
\begin{aligned}
& \left\langle\hat{M}^{\delta}, \hat{M}^{\delta}\right\rangle_{t}=\lambda_{\delta} \int_{0}^{t} \int z^{4} v^{* \delta}(d z) \underset{\delta \rightarrow 0}{\longrightarrow} 3 \sigma^{4} t \\
& \left\langle M^{\delta}, M^{\delta}\right\rangle_{t}=\int_{0}^{t} \int z^{2} v^{* \delta}(d z) \underset{\delta \rightarrow 0}{\longrightarrow} \sigma^{2} t
\end{aligned}
$$

and

$$
\left\langle M^{\delta}, \hat{M}^{\delta}\right\rangle_{t}=\sqrt{\lambda_{\delta}} \int_{0}^{t} \int z^{3} v^{* \delta}(d z) \underset{\delta \rightarrow 0}{\longrightarrow} 0
$$

Now, consider local square-integrable martingales, $M_{\epsilon}^{\delta}$ and $\hat{M}_{\epsilon}^{\delta}$, which include all the jumps of the original martingales in absolute value greater than $\epsilon$, for a given $\epsilon>0$. Their quadratic variations are

$$
\begin{aligned}
& \left\langle M_{\epsilon}^{\delta}, M_{\epsilon}^{\delta}\right\rangle_{t}=\int_{0}^{t} \int_{|z|>\epsilon} z^{2} v^{* \delta}(d z) d s \underset{\delta \rightarrow 0}{\longrightarrow} 0, \\
& \left\langle\hat{M}_{\epsilon}^{\delta}, \hat{M}_{\epsilon}^{\delta}\right\rangle_{t}=\lambda_{\delta} \int_{0}^{t} \int_{|z|>\epsilon} z^{4} v^{* \delta}(d z) d s \underset{\delta \rightarrow 0}{\longrightarrow} 0 .
\end{aligned}
$$

By Rebolledo's theorem (Andersen et al., 1992, p.83), $\left(M^{\delta}, \hat{M}^{\delta}\right) \stackrel{\mathcal{D}}{\longrightarrow}(\sigma B, \xi)$ and therefore, by Proposition VI.3.17 in Jacod and Shiryaev (1987), $\left(\log S^{\delta}, \xi^{\delta}\right) \stackrel{\mathcal{D}}{\longrightarrow}(\log S, \xi)$.

Let us now define $R^{\delta}$ as a process, $\sqrt{\lambda_{\delta}}\left(C(S ., \cdot)-X_{\text {. }}^{\delta}\right)$. In order to see the weak limit of $R^{\delta}$, let us check limits of a few quantities.

$$
\begin{aligned}
\int z v^{* \delta}(d z) & =\theta^{\delta} \underset{\delta \rightarrow 0}{\longrightarrow} \mu-\frac{1}{2} \sigma^{2} \\
\sqrt{\lambda_{\delta}}\left(\int z^{2} v^{* \delta}(d z)-\sigma^{2}\right) & =\sqrt{v_{\delta}}\left(\theta^{\delta}\right)^{2} \underset{\delta \rightarrow 0}{\longrightarrow} 0
\end{aligned}
$$




$$
\begin{aligned}
& \sqrt{\lambda_{\delta}} \int z^{3} v^{* \delta}(d z)=\frac{1}{\sqrt{v_{\delta}}}\left(2\left(\theta^{\delta}\right)^{3}\left(v^{\delta}\right)^{2}+3 \theta^{\delta} \sigma^{2} v^{\delta}\right) \underset{\delta \rightarrow 0}{\longrightarrow} 0 \\
& \lambda_{\delta} \int^{4} z^{* \delta}(d z)=\frac{1}{v^{\delta}}\left(6\left(\theta^{\delta}\right)^{4}\left(v^{\delta}\right)^{3}+12\left(\theta^{\delta}\right)^{2} \sigma^{2}\left(v^{\delta}\right)^{2}+3 \sigma^{4} v^{\delta}\right) \underset{\delta \rightarrow 0}{\longrightarrow} 3 \sigma^{4} \\
& \lambda_{\delta} \int_{|z|>\epsilon} z^{4} v^{* \delta}(d z) \underset{\delta \rightarrow 0}{\longrightarrow} 0 \\
& \lambda_{\delta} \int_{|z|<1} z^{6} v^{* \delta}(d z) \underset{\delta \rightarrow 0}{\longrightarrow} 0 \\
& \lambda_{\delta} \int_{|z|>1} e^{|z|} v^{* \delta}(d z) \underset{\delta \rightarrow 0}{\longrightarrow} 0 .
\end{aligned}
$$

The last convergence is valid as long as $M^{\delta}>1$ and $G^{\delta}>1$, where $M^{\delta}=\left(\sqrt{1 / 4\left(\theta^{\delta}\right)^{2}\left(v^{\delta}\right)^{2}+1 / 2 \sigma^{2} v^{\delta}}+\right.$ $\left.1 / 2 \theta^{\delta} v^{\delta}\right)^{-1}$ and $G^{\delta}=\left(\sqrt{1 / 4\left(\theta^{\delta}\right)^{2}\left(\nu^{\delta}\right)^{2}+1 / 2 \sigma^{2} \nu^{\delta}}-1 / 2 \theta^{\delta} v^{\delta}\right)^{-1}$. Since $M^{\delta} \rightarrow \infty$ and $G^{\delta} \rightarrow \infty$ as $\delta \rightarrow 0$, we can assume that they are less than 1 for sufficiently small $\delta$.

By Itô's formula and Taylor expansion,

$$
\begin{aligned}
R_{t}^{\delta} & =\sqrt{\lambda_{\delta}}\left(C\left(S_{t}^{\delta}, t\right)-C\left(S_{0}, 0\right)-\int_{0}^{t} C_{S}\left(S_{u^{-}}^{\delta}, u\right) d S_{u}^{\delta}\right) \\
& =\sqrt{\lambda_{\delta}}\left(\int_{0}^{t} C_{t}\left(S_{u-}^{\delta}, u\right) d u+\sum_{u \leq t}\left(\Delta C\left(S_{u}^{\delta}, u\right)-C_{S}\left(S_{u-}^{\delta}, u\right) \Delta S_{u}^{\delta}\right)\right) \\
& =\sqrt{\lambda_{\delta}}\left(\int_{0}^{t} C_{t}\left(S_{u-}^{\delta}, u\right) d u+\sum_{u \leq t} \frac{1}{2} C_{S S}\left(S_{u-}^{\delta}, u\right)\left(\Delta S_{u}^{\delta}\right)^{2}+\sum_{u \leq t} \frac{1}{6} C_{S S S}\left(\tilde{Z}_{u}^{\delta}, u\right)\left(\Delta S_{u}^{\delta}\right)^{3}\right),
\end{aligned}
$$

where $\tilde{Z}^{\delta}$ is a process satisfying $\min \left(S_{t-}^{\delta}, S_{t}^{\delta}\right) \leq \tilde{Z}_{t}^{\delta} \leq \max \left(S_{t-}^{\delta}, S_{t}^{\delta}\right)$, for all $0<t<T$. By BlackScholes partial differential equation and using $\Delta S_{t}^{\delta}=S_{t-}^{\delta}\left(e^{\Delta \log S_{t}^{\delta}}-1\right)$,

$$
\begin{aligned}
R_{t}^{\delta}= & \sqrt{\lambda_{\delta}}\left(\int_{0}^{t}-\frac{\sigma^{2}}{2} C_{S S}\left(S_{u-}^{\delta}, u\right)\left(S_{u-}^{\delta}\right)^{2} d u+\sum_{u \leq t} \frac{1}{2} C_{S S}\left(S_{u-}^{\delta}, u\right)\left(\Delta S_{u}^{\delta}\right)^{2}+\sum_{u \leq t} \frac{1}{6} C_{S S S}\left(\tilde{Z}_{u}^{\delta}, u\right)\left(\Delta S_{u}^{\delta}\right)^{3}\right) \\
= & -\frac{\sqrt{\lambda_{\delta}}}{2} \int_{0}^{t} \sigma^{2} C_{S S}\left(S_{u-}^{\delta}, u\right)\left(S_{u-}^{\delta}\right)^{2} d u+\frac{\sqrt{\lambda_{\delta}}}{2} \sum_{u \leq t} C_{S S}\left(S_{u}^{\delta}, u\right)\left(S_{u-}^{\delta}\right)^{2}\left(e^{\Delta \log S_{u}^{\delta}}-1\right)^{2} \\
& +\frac{\sqrt{\lambda_{\delta}}}{6} \sum_{u \leq t} C_{S S S}\left(\tilde{Z}_{u}^{\delta}, u\right)\left(S_{u-}^{\delta}\right)^{3}\left(e^{\Delta \log S_{u}^{\delta}}-1\right)^{3} .
\end{aligned}
$$

We can rewrite $R_{t}^{\delta}$ as

$$
\begin{aligned}
R_{t}^{\delta}= & \frac{1}{2} \int_{0}^{t} C_{S S}\left(S_{u-}^{\delta}, u\right)\left(S_{u}^{\delta}\right)^{2} d \xi_{u}^{\delta} \\
& +\sqrt{\lambda_{\delta}} \sum_{u \leq t} \frac{1}{6}\left(3 C_{S S}\left(S_{u-}^{\delta}, u\right)\left(S_{u-}^{\delta}\right)^{2}+C_{S S S}\left(\tilde{Z}_{u}^{\delta}, u\right)\left(S_{u-}^{\delta}\right)^{3}\right)\left(\Delta Z_{u}^{\delta}\right)^{3} \\
& +\sqrt{\lambda_{\delta}} \sum_{u \leq t} \frac{1}{4}\left(\frac{7}{6} C_{S S}\left(S_{u-}^{\delta}, u\right)\left(S_{u-}^{\delta}\right)^{2}+C_{S S S}\left(\tilde{Z}_{u}^{\delta}, u\right)\left(S_{u-}^{\delta}\right)^{3}\right)\left(\Delta Z_{u}^{\delta}\right)^{4}
\end{aligned}
$$




$$
\begin{gathered}
+\sum_{u \leq t}\left(C_{S S}\left(S_{u-}^{\delta}, u\right)\left(S_{u-}^{\delta}\right)^{2} \times(\text { higher order terms })\right. \\
\left.+C_{S S S}\left(\tilde{Z}_{u}^{\delta}, u\right)\left(S_{u^{-}}^{\delta}\right)^{3} \times(\text { higher order terms })\right)
\end{gathered}
$$

We can also show the following.

(i) By Lemma VI.3.31 in Jacod and Shiryaev (1987) and lemma $1,\left(\log \tilde{Z}^{\delta}, \log S^{\delta}, \xi^{\delta}\right) \stackrel{\mathcal{D}}{\longrightarrow}(\log S$, $\log S, \xi)$, since $\left(\log S^{\delta}, \log S^{\delta}, \xi^{\delta}\right) \stackrel{\mathcal{D}}{\longrightarrow}(\log S, \log S, \xi)$ and $\sup _{t \leq T}\left|\log \tilde{Z}_{t}^{\delta}-\log S_{t}^{\delta}\right|<\sup _{t \leq T}$ $\left|\Delta \log S_{t}^{\delta}\right|=o_{p}(1)$.

(ii) Define $\tilde{M}^{\delta}$ to be $\sqrt{\lambda_{\delta}}\left(\left[\log S^{\delta}, \log S^{\delta}, \log S^{\delta}\right]-\left\langle\log S^{\delta}, \log S^{\delta}, \log S^{\delta}\right\rangle\right)$. Then $\tilde{M}^{\delta}$ is a squareintegrable martingale and

$$
\begin{aligned}
\left\langle\tilde{M}^{\delta}, \tilde{M}^{\delta}\right\rangle_{t} & =\lambda_{\delta} \int_{0}^{t} \int z^{6} v^{* \delta}(d z) d s \\
& =\lambda_{\delta} \int_{0}^{t} \int_{|z|>1} z^{6} v^{* \delta}(d z) d s+\lambda_{\delta} \int_{0}^{t} \int_{|z|<1} z^{6} v^{* \delta}(d z) d s \underset{\delta \rightarrow 0}{\longrightarrow} 0,
\end{aligned}
$$

which can be shown by (B.1). Also define $\tilde{M}_{\epsilon}^{\delta}$ to be the martingale part of $\sqrt{\lambda_{\delta}}\left[\log S^{\delta}, \log S^{\delta}, \log S^{\delta}\right]$ with jump size greater than $\epsilon$ in absolute value. Then,

$$
\left\langle\tilde{M}_{\epsilon}^{\delta}, \tilde{M}_{\epsilon}^{\delta}\right\rangle_{t}=\lambda_{\delta} \int_{0}^{t} \int_{|z|>\epsilon} z^{6} v^{* \delta}(d z) d s \leq \lambda_{\delta} \int_{0}^{t} \int z^{6} v^{* \delta}(d z) d s \underset{\delta \rightarrow 0}{\longrightarrow} 0 .
$$

By Rebolledo's theorem (Andersen et al., 1992), $\tilde{M}^{\delta} \stackrel{\mathcal{D}}{\longrightarrow} 0$ and thus, $\sqrt{\lambda_{\delta}} \sum_{u \leq t}\left(\Delta Z_{u}^{\delta}\right)^{3} \stackrel{\mathcal{D}}{\longrightarrow} 0$ since $\sqrt{\lambda_{\delta}} \int_{0}^{t} \int z^{3} v^{* \delta}(d z) d s \rightarrow 0$.

(iii) Since $\sum_{u \leq t} \sqrt{\lambda_{\delta}}\left(\Delta Z_{u}^{\delta}\right)^{4} \geq 0$ and $E\left(\sum_{u \leq t} \sqrt{\lambda_{\delta}}\left(\Delta Z_{u}^{\delta}\right)^{4}\right)=\sqrt{\lambda_{\delta}} \int_{0}^{t} \int z^{4} v^{* \delta}(d z) d s \underset{\delta \rightarrow 0}{\longrightarrow} 0$, we can see easily that $\sum_{u \leq t} \sqrt{\lambda_{\delta}}\left(\Delta Z_{u}^{\delta}\right)^{4} \stackrel{\mathcal{D}}{\longrightarrow} 0$.

(iv) For higher order terms of $\sum_{u \leq t} \sqrt{\lambda_{\delta}}\left(\Delta Z_{u}^{\delta}\right)^{m}, m=5,6, \ldots$, we can show that $\sum_{u \leq t} \sqrt{\lambda_{\delta}}\left(\Delta Z_{u}^{\delta}\right)^{2 k} \stackrel{\mathcal{D}}{\longrightarrow} 0$ and $\sum_{u \leq t} \sqrt{\lambda_{\delta}}\left(\Delta Z_{u}^{\delta}\right)^{2 k-1} \stackrel{\mathcal{D}}{\longrightarrow} 0$ for $k \geq 3$, separately as follows.

$$
\begin{aligned}
E\left(\sum_{u \leq t} \sqrt{\lambda_{\delta}}\left(\Delta Z_{u}^{\delta}\right)^{2 k}\right) & =\frac{1}{\sqrt{\lambda_{\delta}}} \lambda_{\delta} t \int z^{2 k} v^{* \delta}(d z) \\
& =\frac{1}{\sqrt{\lambda_{\delta}}}\left(\lambda_{\delta} t \int_{|z|<1} z^{2 k} v^{* \delta}(d z)+\lambda_{\delta} t \int_{|z|>1} z^{2 k} v^{* \delta}(d z)\right) \\
& \leq \frac{1}{\sqrt{\lambda_{\delta}}}\left(\lambda_{\delta} t \int_{|z|<1} z^{4} v^{* \delta}(d z)+\lambda_{\delta} t \int_{|z|>1} z^{2 k} v^{* \delta}(d z)\right) \\
& \longrightarrow 0
\end{aligned}
$$




$$
\begin{aligned}
E\left(\sum_{u \leq t} \sqrt{\lambda_{\delta}}\left|\Delta Z_{u}^{\delta}\right|^{2 k-1}\right) & =\frac{1}{\sqrt{\lambda_{\delta}}} \lambda_{\delta} t \int|z|^{2 k-1} v^{* \delta}(d z) \\
& =\frac{1}{\sqrt{\lambda_{\delta}}}\left(\lambda_{\delta} t \int_{|z|<1}|z|^{2 k-1} v^{* \delta}(d z)+\lambda_{\delta} t \int_{|z|>1}|z|^{2 k-1} v^{* \delta}(d z)\right) \\
& \leq \frac{1}{\sqrt{\lambda_{\delta}}}\left(\lambda_{\delta} t \int_{|z|<1} z^{4} v^{* \delta}(d z)+\lambda_{\delta} t \int_{|z|>1}|z|^{2 k-1} v^{* \delta}(d z)\right) \\
& \longrightarrow 0 .
\end{aligned}
$$

(v) By Theorem 2.7 in Kurtz and Protter (1991) and (i) through (iv) above,

$$
\begin{aligned}
& \left(\log \tilde{Z}^{\delta}, \log S^{\delta}, \xi^{\delta}, \frac{1}{2} \int_{0}\left(S_{u-}^{\delta}\right)^{2} C_{S S}\left(S_{u-}^{\delta}, u\right) d \xi_{u}^{\delta},\right. \\
& \sqrt{\lambda_{\delta}} \sum_{u \leq \cdot} \frac{1}{6}\left(3 C_{S S}\left(S_{u-}^{\delta}, u\right)\left(S_{u-}^{\delta}\right)^{2}+C_{S S S}\left(\tilde{Z}_{u}^{\delta}, u\right)\left(S_{u-}^{\delta}\right)^{3}\right)\left(\Delta Z_{u}^{\delta}\right)^{3}, \\
& \left.\sqrt{\lambda_{\delta}} \sum_{u \leq .} \frac{1}{4}\left(\frac{7}{6} C_{S S}\left(S_{u-}^{\delta}, u\right)\left(S_{u-}^{\delta}\right)^{2}+C_{S S S}\left(\tilde{Z}_{u}^{\delta}, u\right)\left(S_{u-}^{\delta}\right)^{3}\right)\left(\Delta Z_{u}^{\delta}\right)^{4}\right) \\
& \stackrel{\mathcal{D}}{\longrightarrow}\left(\log S, \log S, \xi, \frac{1}{2} \int_{0} S_{u}^{2} C_{S S}\left(S_{u}, u\right) d \xi_{u}, 0,0\right) .
\end{aligned}
$$

For this, we check conditions including the followings.

- For $\xi^{\delta}$

$$
\begin{aligned}
\xi_{t}^{\delta} & =\sqrt{\lambda_{\delta}}\left(\left[\log S^{\delta}, \log S^{\delta}\right]_{t}-\left\langle\log S^{\delta}, \log S^{\delta}\right\rangle_{t}\right)+\sqrt{\lambda_{\delta}}\left(\left\langle\log S^{\delta}, \log S^{\delta}\right\rangle_{t}-\sigma^{2} t\right) \\
& =: M_{\xi, t}^{\delta}+A_{\xi, t}^{\delta},
\end{aligned}
$$

$E\left[M_{\xi}^{\delta}, M_{\xi}^{\delta}\right]_{t}<\infty$ and $\sup _{\delta} E\left(M_{\xi, t}^{\delta}+T_{t}\left(A_{\xi}^{\delta}\right)\right)<\infty$, where $T_{t}\left(A_{\xi}^{\delta}\right)$ is the total variation of $A_{\xi}^{\delta}$ on $[0, t]$.

- For $\sqrt{\lambda_{\delta}} \sum_{u \leq t}\left(\Delta Z_{u}^{\delta}\right)^{3}$

$$
\begin{aligned}
\sqrt{\lambda_{\delta}} \sum_{u \leq t}\left(\Delta Z_{u}^{\delta}\right)^{3}= & \sqrt{\lambda_{\delta}}\left(\left[\log S^{\delta}, \log S^{\delta}, \log S^{\delta}\right]_{t}-\left\langle\log S^{\delta}, \log S^{\delta}, \log S^{\delta}\right\rangle_{t}\right) \\
& +\sqrt{\lambda_{\delta}}\left\langle\log S^{\delta}, \log S^{\delta}, \log S^{\delta}\right\rangle_{t} \\
=: & M_{(\Delta \log S)^{3}, t}^{\delta}+A_{(\Delta \log S)^{3}, t}^{\delta},
\end{aligned}
$$

$E\left[M_{(\Delta \log S)^{3}}^{\delta}, M_{(\Delta \log S)^{3}}^{\delta}\right]_{t}<\infty$ and $\sup _{\delta} E\left(M_{(\Delta \log S)^{3}, t}^{\delta}+T_{t}\left(A_{(\Delta \log S)^{3}}^{\delta}\right)\right)<\infty$, where $T_{t}\left(A_{(\Delta \log S)^{3}}^{\delta}\right)$ is the total variation of $A_{(\Delta \log S)^{3}}^{\delta}$ on $[0, t]$.

- For $\sqrt{\lambda_{\delta}} \sum_{u \leq t}\left(\Delta \log S_{u}^{\delta}\right)^{4}$,

$$
\sqrt{\lambda_{\delta}} \sum_{u \leq t}\left(\Delta \log S_{u}^{\delta}\right)^{4}=\sqrt{\lambda_{\delta}}\left(\left[\log S^{\delta}, \log S^{\delta}, \log S^{\delta}, \log S^{\delta}\right]_{t}-\left\langle\log S^{\delta}, \log S^{\delta}, \log S^{\delta}, \log S^{\delta}\right\rangle_{t}\right)
$$




$$
\begin{aligned}
& +\sqrt{\lambda_{\delta}}\left\langle\log S^{\delta}, \log S^{\delta}, \log S^{\delta}, \log S^{\delta}\right\rangle_{t} \\
=: & M_{(\Delta \log S)^{4}, t}^{\delta}+A_{(\Delta \log S)^{4}, t^{\prime}}^{\delta}
\end{aligned}
$$

$E\left[M_{(\Delta \log S)^{4}}^{\delta}, M_{(\Delta \log S)^{4}}^{\delta}\right]_{t}<\infty$ and $\sup _{\delta} E\left(M_{(\Delta \log S)^{4}, t}^{\delta}+T_{t}\left(A_{(\Delta \log S)^{4}}^{\delta}\right)\right)<\infty$, where $T_{t}\left(A_{(\Delta \log S)^{4}}^{\delta}\right)$ is the total variation of $A_{(\Delta \log S)^{4}}^{\delta}$ on $[0, t]$.

(iv) The last term in (B.3) has a higher order than

$$
\sqrt{\lambda_{\delta}} \sum_{u \leq} \frac{1}{4}\left(\frac{7}{6} C_{S S}\left(S_{u-}^{\delta}, u\right)\left(S_{u-}^{\delta}\right)^{2}+C_{S S S}\left(\tilde{Z}_{u}^{\delta}, u\right)\left(S_{u-}^{\delta}\right)^{3}\right)\left(\Delta \log S_{u}^{\delta}\right)^{4} .
$$

Therefore,

$$
R_{t}^{\delta} \stackrel{\mathcal{D}}{\longrightarrow} \int_{0}^{t} \frac{1}{2} S_{u}^{2} C_{S S}\left(S_{u}, u\right) d \xi_{u},
$$

as a process jointly with $S^{\delta}$.

\section{Appendix C: Proof of Proposition 2}

We know from Theorem $1,\left(R^{\delta}, S^{\delta}\right)$ converges to $(R, S)$ weakly, and by assumption, we have

$$
\left(R^{\delta}, S^{\delta}, \eta^{\delta}\right) \stackrel{\mathcal{D}}{\longrightarrow}(R, S, \eta) .
$$

$S^{\delta}, R^{\delta}$ and $\eta^{\delta}$ are adapted to the filtration generated by $S^{\delta}$. In order to prove this proposition, we only need to check if $S^{\delta}$ satisfies the proper conditions as an integrator of the stochastic integral, given in Kurtz and Protter (1991).

Proof: $S^{\delta}, R^{\delta}, \eta^{\delta}$ are adapted to the filtration generated by $S^{\delta}$, cádlág processes, and $S^{\delta}$ is a semimartingale. Since $S_{t}^{\delta}=S_{0} e^{Z_{t}^{\delta}}$ and $Z_{t}^{\delta}=\theta^{\delta} t+\int_{0}^{t} \int z\left(\mu^{\delta}(d s, d z)-v^{* \delta}(d z) d s\right)$,

$$
\begin{aligned}
S_{t}^{\delta} & =\int_{0}^{t} S_{u-}^{\delta} d Z_{u}^{\delta}+\sum_{u \leq t} S_{u-}^{\delta}\left(e^{\Delta Z_{u}^{\delta}}-1-\Delta Z_{u}^{\delta}\right) \\
& =\int_{0}^{t} \theta^{\delta} S_{u-}^{\delta} d u+\int_{0}^{t} S_{u-}^{\delta} \int z\left(\mu^{\delta}(d u, d z)-v^{* \delta}(d z) d u\right)+\int_{0}^{t} S_{u^{-}}^{\delta} \int\left(e^{z}-1-z\right) \mu^{\delta}(d u, d z) \\
& =\int_{0}^{t} S_{u-}^{\delta} \int\left(e^{z}-1\right)\left(\mu^{\delta}(d u, d z)-v^{* \delta}(d z) d u\right)+\int_{0}^{t} \theta^{\delta} S_{u-}^{\delta} d u+\int_{0}^{t} S_{u-}^{\delta} \int\left(e^{z}-1-z\right) v^{* \delta}(d z) d u \\
& =: M_{(S), t}^{\delta}+A_{(S), t}^{\delta},
\end{aligned}
$$

where $M_{(S)}^{\delta}$ and $A_{(S)}^{\delta}$ are the martingale and finite variation parts of $S^{\delta}$, respectively. Then,

$$
\begin{aligned}
E\left[M_{(S)}^{\delta}, M_{(S)}^{\delta}\right]_{t} & =E\left(\sum_{u \leq t}\left(S_{u-}^{\delta}\right)^{2}\left(e^{\Delta Z_{u}^{\delta}}-1\right)^{2}\right)=E\left(\int_{0}^{t}\left(S_{u-}^{\delta}\right)^{2} d u\right) \int\left(e^{z}-1\right)^{2} v^{* \delta}(d z) \\
E\left(T_{t}\left(A_{(S)}^{\delta}\right)\right) & =\int_{0}^{t} E\left(S_{u-}^{\delta}\right) d u \times\left|\int\left(e^{z}-1\right) v^{* \delta}(d z)\right|
\end{aligned}
$$


where $T_{t}\left(A_{(S)}^{\delta}\right)$ is the total variation of $A_{(S)}^{\delta}$ on $[0, t]$. Using the fact that $Z_{t}^{\delta}$ is written as $\theta^{\delta} G_{t}^{\delta}+\sigma^{\delta} W_{G_{t}^{\delta}}$ where $W$ is a standard Brownian motion and $G^{\delta}$ is a Gamma process with $\left(1 / v^{\delta}, v^{\delta}\right)$, we can see that as $\delta \rightarrow 0, E\left(S_{t}^{\delta}\right) \rightarrow S_{0} e^{\mu t}$ and $E\left(S_{t}^{\delta}\right)^{2} \rightarrow S_{0}^{2} e^{\left(2 \mu+\sigma^{2}\right) t} . E\left[M_{(S)}^{\delta}, M_{(S)}^{\delta}\right]_{t}<\infty$ and $\sup _{\delta} E\left(M_{(S), t}^{\delta}+T_{t}\left(A_{(S)}^{\delta}\right)\right)<$ $\infty$, since $\int\left(e^{z}-1\right) v^{* \delta}(d z)$ and $\int\left(e^{z}-1\right)^{2} v^{* \delta}(d z)$ can be bounded as $\delta \rightarrow 0$. Therefore, by Theorem 2.7 in Kurtz and Protter $(1991),\left(R^{\delta}, S^{\delta}, \eta^{\delta}, \int \eta^{\delta} d S^{\delta}\right) \stackrel{\mathcal{D}}{\longrightarrow}\left(R, S, \eta, \int \eta d S\right)$ and thus, $\left(R^{\delta}, S^{\delta}, K^{\delta}\right) \stackrel{\mathcal{D}}{\longrightarrow}$ $(R, S, K)$.

\section{References}

Andersen, P. K., Borgen, O., Gill, R. D. and Keiding, N. (1992). Statistical Models Based on Counting Processes, Springer-Verlag, New York.

Barndorff-Nielsen, O. E. (1998). Processes of normal inverse Gaussian type, Finance and Stochastics, 2, 41-68.

Carr, P., Geman, H., Madan, D. B. and Yor, M. (2002). The fine structure of asset returns: An empirical investigation, Journal of Business, 75, 305-332.

Cont, R. and Tankov, P. (2004). Financial Modelling with Jump Processes, Chapman \& Hall/CRC.

Eberlein, E. and Keller, U. (1995). Hyperbolic distributions in Finance, Bernoulli, 1, 281-299.

Geman, H. (2002). Pure jump Lévy processes for asset price modelling, Journal of Banking and Finance, 21, 755-763.

Hong, D. and Wee, I. (2003). Convergence of Jump-Diffusion models to the Black-Scholes model, Stochastic Analysis and Applications, 21, 141-160.

Jacod, J. and Shiryaev, A. N. (1987). Limit Theorems for Stochastic Processes, Springer-Verlag, Berlin.

Kurtz, T. G. and Protter, P. E. (1991). Weak limit theorems for stochastic integrals and stochastic differential equations, Annals of Probability, 19, 1035-1070.

Madan, D. B. and Seneta, E. (1990). The VG model for share market returns, Journal of Business, 63, 511-524.

Schoutens, W. (2003). Lévy Processes in Finance: Pricing Financial Derivatives, Wiley.

Song, S. and Mykland, P. A. (2006). An asymptotic decomposition of hedging errors, Journal of the Korean Statistical Society, 35, 115-142.

Song, S. and Song, J. (2008). Asymptotic option price with bounded expected loss, Journal of the Korean Statistical Society, 37, 323-334. 